\title{
Molecular genetics of naringenin biosynthesis, a typical plant secondary metabolite produced by Streptomyces clavuligerus
}

\author{
Rubén Álvarez-Álvarez ${ }^{1,2}$, Alma Botas ${ }^{2}$, Silvia M. Albillos², Angel Rumbero ${ }^{3}$, Juan F. Martín ${ }^{1}$ and Paloma Liras ${ }^{1 *}$
}

\begin{abstract}
Background: Some types of flavonoid intermediates seemed to be restricted to plants. Naringenin is a typical plant metabolite, that has never been reported to be produced in prokariotes. Naringenin is formed by the action of a chalcone synthase using as starter 4-coumaroyl-CoA, which in dicotyledonous plants derives from phenylalanine by the action of a phenylalanine ammonia lyase.

Results: A compound produced by Streptomyces clavuligerus has been identified by LC-MS and NMR as naringenin and coelutes in HPLC with a naringenin standard. Genome mining of S. clavuligerus revealed the presence of a gene for a chalcone synthase (ncs), side by side to a gene encoding a P450 cytochrome (ncyP) and separated from a gene encoding a Pal/Tal ammonia lyase (tal). Deletion of any of these genes results in naringenin non producer mutants. Complementation with the deleted gene restores naringenin production in the transformants. Furthermore, naringenin production increases in cultures supplemented with phenylalanine or tyrosine.
\end{abstract}

Conclusion: This is the first time that naringenin is reported to be produced naturally in a prokariote. Interestingly three non-clustered genes are involved in naringenin production, which is unusual for secondary metabolites. A tentative pathway for naringenin biosynthesis has been proposed.

Keywords: Naringenin, Secondary metabolism, Streptomyces clavuligerus, Histidine/phenylalanine ammonium lyase

\section{Background}

S. clavuligerus ATCC 27064 is an industrially relevant actinobacteria, used for the production of clavulanic acid (CA) [1]. This strain has a peculiar carbohydrate metabolism [2] and is able to produce in addition to CA other bioactive compounds such as cephamycin C [3], clavams $[4]$ and holomycin [5, 6]. Interestingly, sequencing of the S. clavuligerus genome revealed that this organism has potential ability to produce a wealth of additional secondary metabolites [7]. Up to 25 clusters for putative secondary metabolites are located in the $6.8 \mathrm{Mb}$ chromosome

\footnotetext{
*Correspondence: paloma.liras@unileon.es

${ }^{1}$ Microbiology Section, Department of Molecular Biology, Faculty of Biology and Environmental Sciences, Vegazana Campus, University of León, León 24071, Spain

Full list of author information is available at the end of the article
}

of $S$. clavuligerus and 23 more are present in the $1.8 \mathrm{Mb}$ megaplasmid pSCL4 $[7,8]$. They include ten clusters containing genes for nonribosomal peptide synthetases (NRPS), six clusters with genes for polyketide synthases (PKS), as well as NRPS-PKS hybrid clusters and genes for putative terpene synthesizing enzymes.

Gene clusters encoding novel bioactive products are of utmost interest. The homology of some clusters with previously studied orthologous in other Streptomyces species suggest that $S$. clavuligerus might produce staurosporine, moenomycin, indigoidine, and enediyne-like compounds, some of which are antitumor agents. Since most of these compounds have never been detected in S. clavuligerus cultures they must be silent clusters that are only expressed in response to specific nutritional or physiological signals, or in response to elicitors $[9,10]$. 
Chalcone synthases are type III polyketide synthases involved in the condensation of different CoA-activated precursor starter units (i.e. phenylpropenoids) to produce flavonoid-type chalcones. They are very frequent in fungi and in plants, where they serve as precursors of different types of flavonoids [11] but are rare in actinomycetes. Some types of flavonoid intermediates, e.g. naringenin, appeared to be restricted to plants. An interesting question is whether there are chemical structures of secondary metabolites restricted to plants or if many of them can be found in some actinobacteria. In this work we describe that $S$. clavuligerus is able to produce naringenin, a flavanone important as antioxidant [12], described to act as antiinflamatory, chemoprotective and antitumor agent $[13,14]$.
Naringenin is naturally produced in several plants, especially in grapefruit, and its biosynthesis has been studied in Medicago, parsley and other plants (Fig. 1) but it is unknown if the same pathway occurs in S. clavuligerus. The starter unit for naringenin biosynthesis is 4-coumaroyl-CoA, which in dicotyledonous plants derives from phenylalanine by the action of a phenylalanine ammonia lyase (PAL). This compound is hydroxylated at carbon 4 by a cinnamate-4-hydroxylase and activated by a CoA-dependent ligase [15]. Monocotyledonous plants might use, in addition, tyrosine as substrate producing directly $p$-coumaric acid without the need of the cinnamate-4-hydroxylase. A related enzyme, encoded by the encP gene has been detected in Streptomyces maritimus and found to convert phenylalanine in cinnamic acid, required for enterocin biosynthesis [16],

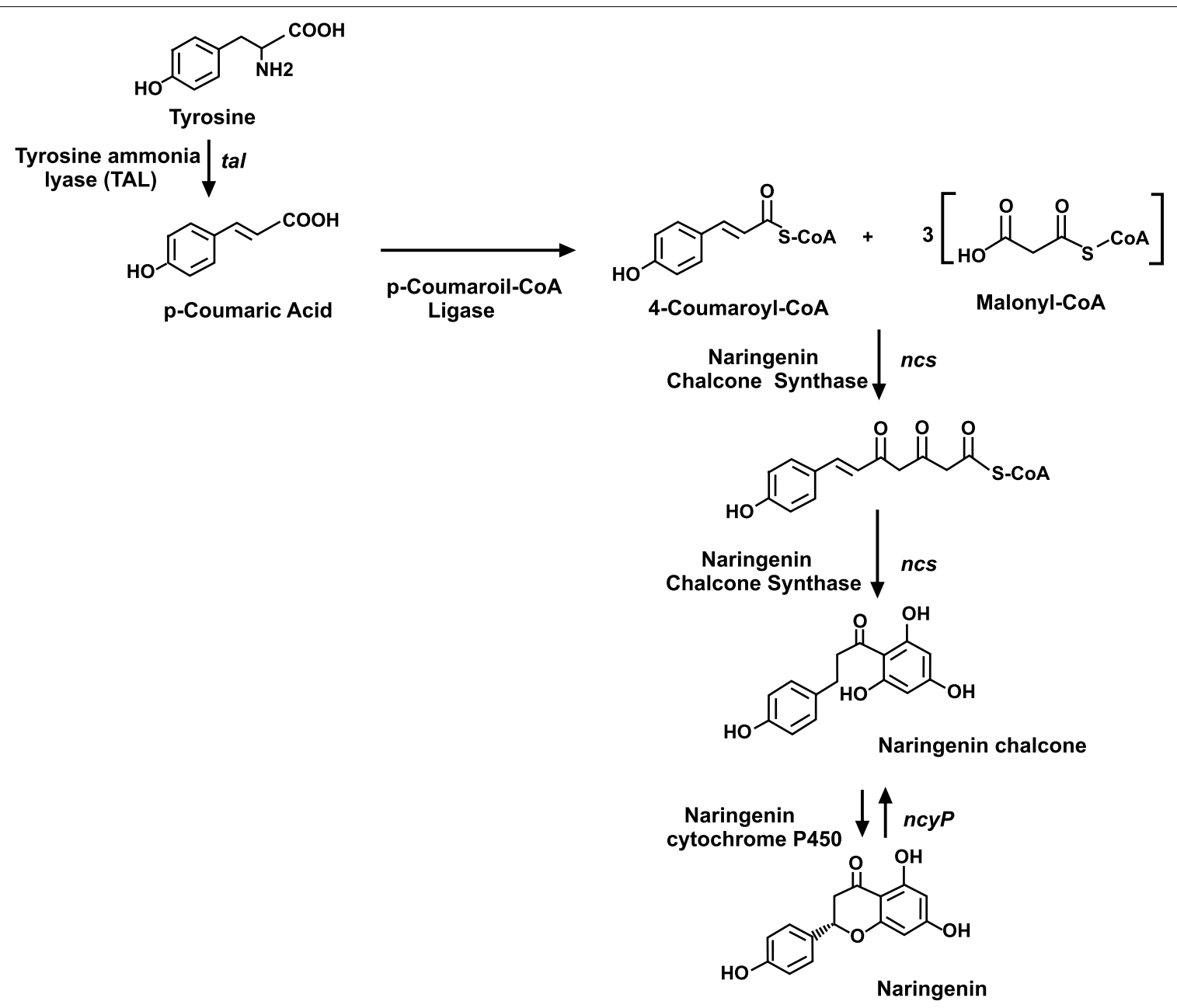

Fig. 1 Proposed naringenin biosynthesis pathway in S. clavuligerus. The names of the intermediate compounds, enzymes involved and genes are indicated. Naringenin biosynthesis starter in plants is usually phenylalanine and there are additional steps for the hydroxylation of cinnamic acid to produce $p$-coumaroic acid 
which lacks the hydroxyl group at the $\mathrm{C}-4$ position, but production of naringenin in prokaryotes has never been reported. It was therefore of interest to study if the naringenin pathway and putative gene cluster(s) in S. clavuligerus is similar to that of plants and whether it is expressed under some specific conditions.

\section{Results}

\section{Characterization of naringenin as a novel product of $S$.} clavuligerus ATCC 27064

In the course of a comparative HPLC study of the compounds produced by S. clavuligerus in TSB and SA media a large absorbance peak was found in cultures grown in TSB medium that was not present in the broth of SA grown cultures. TSB is a tryptic hydrolysate of soybean meal rich in plant-derived amino acids whereas SA contains only asparagine as nitrogen source. The peak eluted at a retention time of 6 min under the HPLC conditions used in the assay (Fig. 2) and corresponds to an extracellular compound that is also present in extracts of washed

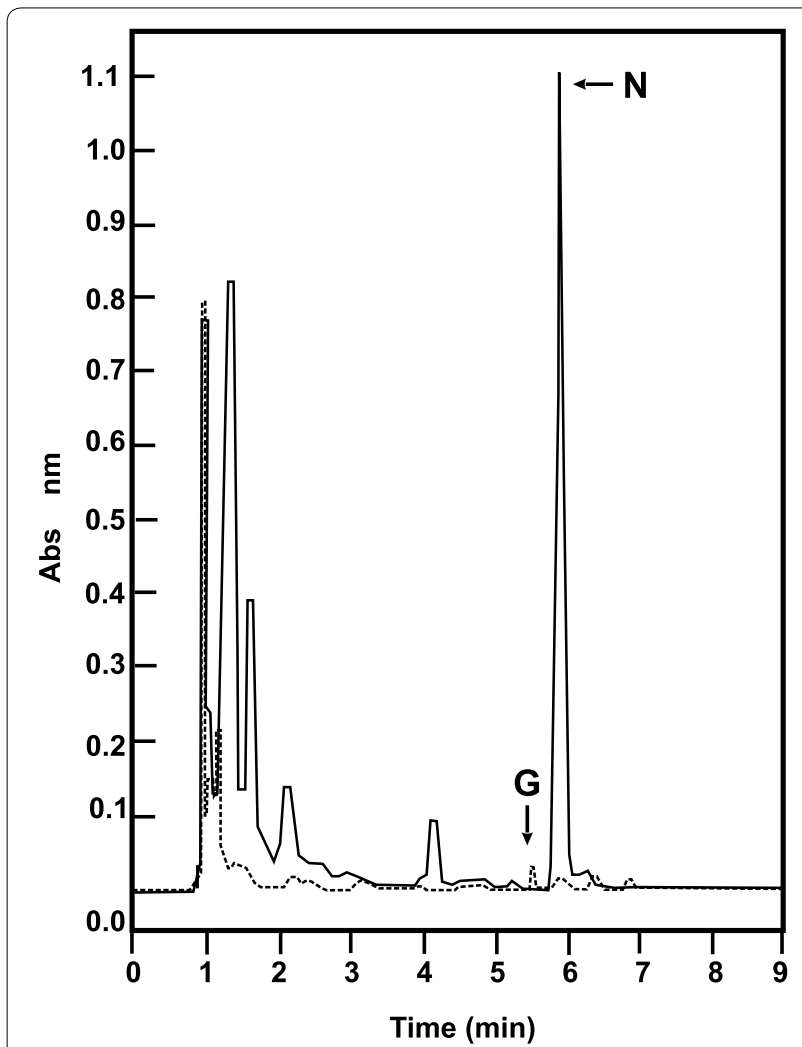

Fig. $2 \mathrm{HPLC}$ analysis of culture broths of S. clavuligerus. The broth of cultures grown in SA medium (discontinuous lane) and TSB medium (continuous line) were analyzed as indicated in "Methods". The peak with a retention time of 6.0 min coincides with pure commercial naringenin $(\mathrm{N})$. Pure genistein $(\mathrm{G})$ used as standard has a retention time of $5.4 \mathrm{~min}$ cells at about $1 \%$ of the concentration found in the broth. No appreciable amount of the compound was found in the unseeded TSB medium indicating that it is formed de novo in S. clavuligerus cultures.

To purify the compound batch cultures were carried out for $72 \mathrm{~h}$ to obtain a total of $2.4 \mathrm{l}$ of culture broth. The centrifuged culture supernatant was concentrated under vacuum and extracted with ethyl acetate as indicated in "Methods". After repeated HPLC injections the compound eluting at $6.0 \mathrm{~min}$ was collected and lyophilized to obtain $5 \mathrm{mg}$ of pure material that was used for the chemical characterization of the molecule.

\section{The compound produced by S. clavuligerus as identified} by LC-MS and NMR is naringenin and coelutes in HPLC with authentic naringenin

A mass $[\mathrm{M}+\mathrm{H}]^{+}$of 273 and a molecular formula of $\mathrm{C}_{15} \mathrm{H}_{12} \mathrm{O}_{5}$ was obtained from LC-MS and from the ${ }^{13} \mathrm{C}$ NMR analysis. Examination of NMR data analysis (Fig. 3) suggested that compound 1 was a flavanone. Its ${ }^{1} \mathrm{H}$-NMR spectrum revealed characteristic resonances of aromatic protons including four proton signals of the B-ring appearing as two doublets at $\delta 7.37$ and $6.87 \mathrm{ppm}$ with $\mathrm{J}=8.6 \mathrm{~Hz}$ due to ortho- coupling that were assigned to $\mathrm{H}-2^{\prime}, 6^{\prime}$ and $\mathrm{H}-3^{\prime}, 5$, respectively; the two meta-coupled protons at $\delta 5.95$ and $5.94 \mathrm{ppm}$ with $\mathrm{J}=2.0 \mathrm{~Hz}$ were assigned to $\mathrm{H}-6$ and $\mathrm{H}-8$. The presence of the quelated signal of 5-OH with the 4-keto group at $\delta 12.15$ and signals at $\delta 5.40(1 \mathrm{H}, \mathrm{dd}, \mathrm{J}=13.1$ and $3.0 \mathrm{~Hz}), \delta 2.70(1 \mathrm{H}$, $\mathrm{dd}, \mathrm{J}=17.2$ and $3.0 \mathrm{~Hz})$ and $\delta 3.15(1 \mathrm{H}, \mathrm{dd}, \mathrm{J}=17.2$ and $13.1 \mathrm{~Hz}$ ), in conjunction with the ${ }^{13} \mathrm{C}$-NMR signals at $\delta$ 79.04 and $42.66 \mathrm{ppm}$, inferred from HMQC spectrum, pointed to the presence of an $-\mathrm{O}-\mathrm{CH}-\mathrm{CH}_{2}-\mathrm{CO}-$ system in the $\mathrm{C}$-ring of the flavanone component. This conclusion was reinforced by the correlation of peak signals at $\delta 3.15$ and $2.70 \mathrm{ppm}$ with the signal at $\delta 5.40$ observed in the ${ }^{1} \mathrm{H}-{ }^{1} \mathrm{H}$ COSY spectrum.

The assignment of the signals of the ${ }^{13} \mathrm{C}$-NMR spectrum, corresponding at $\delta 95.00(\mathrm{C}-6), \delta 95.8(\mathrm{C}-8), \delta$ $115.22\left(\mathrm{C}-3^{\prime}\right.$ and $\left.\mathrm{C}-5^{\prime}\right)$ and $\delta 128.10\left(\mathrm{C}-2^{\prime}\right.$ and $\left.\mathrm{C}-6^{\prime}\right)$ was carried out by using the HSQC-HMQC spectrum, which showed a correlation peak via ${ }^{1} \mathrm{~J}_{\mathrm{C}-\mathrm{H}}$. Other heteronuclear correlations deduced from the HMBC spectrum (Fig. 3) via ${ }^{2} \mathrm{~J}_{\mathrm{C}-\mathrm{H}}$ and ${ }^{3} \mathrm{~J}_{\mathrm{C}-\mathrm{H}}$, were used to assign the signals corresponding at $\delta 196.26(\mathrm{C}-4), \delta 157.82\left(\mathrm{C}-4^{\prime}\right), \delta 166.59$ (C-7), $\delta 164.11$ (C-5), $\delta 163.50$ (C-8a), $\delta 129.49\left(\mathrm{C}-1^{\prime}\right)$ and $\delta 102.19$ (C-4a).

All these NMR data together with the mass spectrum suggested the structure of naringenin [17] for compound 1. The produced compound was different from genistein, a plant flavonoid intermediate similar to naringenin but carrying an additional double bond; both compounds 


\section{a Heteronuclear multiple-bond correlation (HMBC) for compound 1}

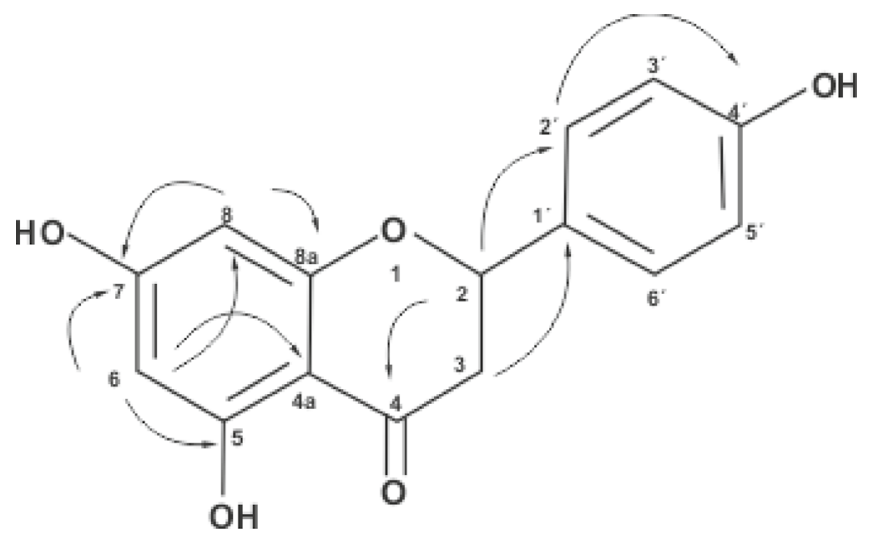

b ${ }^{1} \mathrm{H}$ and ${ }^{13} \mathrm{C}-\mathrm{NMR}$ chemical shifts assignments for naringenine (1) in acetone- $\mathrm{d}_{6}$

\begin{tabular}{|c|c|c|}
\hline $\mathbf{N}^{\mathbf{0}}$ & ${ }^{13} \mathbf{C}$ & ${ }^{{ }^{1} \mathbf{H}}$ \\
\hline $\mathbf{2}$ & 79.04 & $5.40\left(\mathrm{dd}, \mathrm{J}_{2,3 b}=13.1 ; \mathrm{J}_{2,3 a}=3.0 \mathrm{~Hz}\right)$ \\
\hline $\mathbf{3}$ & 42.66 & $\begin{array}{l}\mathrm{H}-3 a: 2.70\left(\mathrm{dd}, \mathrm{J}_{3 a, 3 b}=17.2 ; \mathrm{J}_{3 a, 2}=3.0 \mathrm{~Hz}\right) \\
\mathrm{H}-3 \mathrm{~b}: 3.15\left(\mathrm{dd}, \mathrm{J}_{3 b, 3 a}=17.2 ; \mathrm{J}_{3 b, 2}=13.1 \mathrm{~Hz}\right)\end{array}$ \\
\hline $\mathbf{4}$ & 196.26 & \\
\hline $\mathbf{4 a}$ & 102.19 & \\
\hline $\mathbf{5}$ & 164.11 & $5.95\left(\mathrm{~d}, \mathrm{~J}_{6,8}=2.0 \mathrm{~Hz}\right)$ \\
\hline $\mathbf{6}$ & 95.00 & \\
\hline $\mathbf{7}$ & 166.59 & $5.94\left(\mathrm{~d}, \mathrm{~J}_{8,6}=2.0 \mathrm{~Hz}\right.$ \\
\hline $\mathbf{8}$ & 95.80 & \\
\hline $\mathbf{8 a}$ & 163.50 & \\
\hline $\mathbf{1}^{\prime}$ & 129.49 & $7.37\left(\mathrm{~d}, \mathrm{~J}_{2^{\prime}, 3^{\prime}}=\mathrm{J}_{6^{\prime}, 5^{\prime}}=8.6 \mathrm{~Hz}\right)$ \\
\hline $\mathbf{2}^{\prime}, \mathbf{6}^{\prime}$ & 128.10 & $\left.6.87\left(\mathrm{~d}, \mathrm{~J}_{3^{\prime}, 2^{\prime}}=\mathrm{J}_{5^{\prime}, 6^{\prime}}=8.6 \mathrm{~Hz}\right)\right)$ \\
\hline $\mathbf{3}^{\prime}, \mathbf{5}^{\prime}$ & 115.22 & \\
\hline $\mathbf{4}$ & 157.82 & $12.15 \mathrm{~s}$ \\
\hline $\mathbf{5 - O H}$ & & \\
\hline
\end{tabular}

Fig. 3 NMR analysis for the purified compound. a Heteronuclear multiple-bond correlation. $\mathbf{b}^{1} \mathrm{H}$ and ${ }^{13} \mathrm{C}-\mathrm{NMR}$ chemical shifts assignments for naringenin in acetone-d6

were clearly resolved by HPLC (retention times of 5.4 and $6.0 \mathrm{~min}$ for genistein and naringenin, respectively).

\section{Production of naringenin is stimulated by aromatic amino acids}

Production of naringenin in TSB medium increases in parallel to the growth of the culture reaching the maximum level at $84 \mathrm{~h}$ (Fig. 4). TSB is a complex medium; however, it might not have the adequate precursor requirements for naringenin production. Since this compound is known to be formed from malonate and tyrosine or phenylalanine in different plants, we supplemented the TSB medium separately with different organic acids (acetic, butyric, palmitic and oleic acid, as sodium salts) at $5 \mathrm{mM}$ concentration and the final production of naringenin was measured in each case. Acetate and oleic acid did not improve naringenin production, but butyrate and palmitic acid improved it 1.15and 1.63 -fold, respectively.

In addition, cultures were supplemented with phenylalanine or tyrosine at 5 and $10 \mathrm{mM}$ concentration (Fig. 4c). Tyrosine improved the naringenin production 


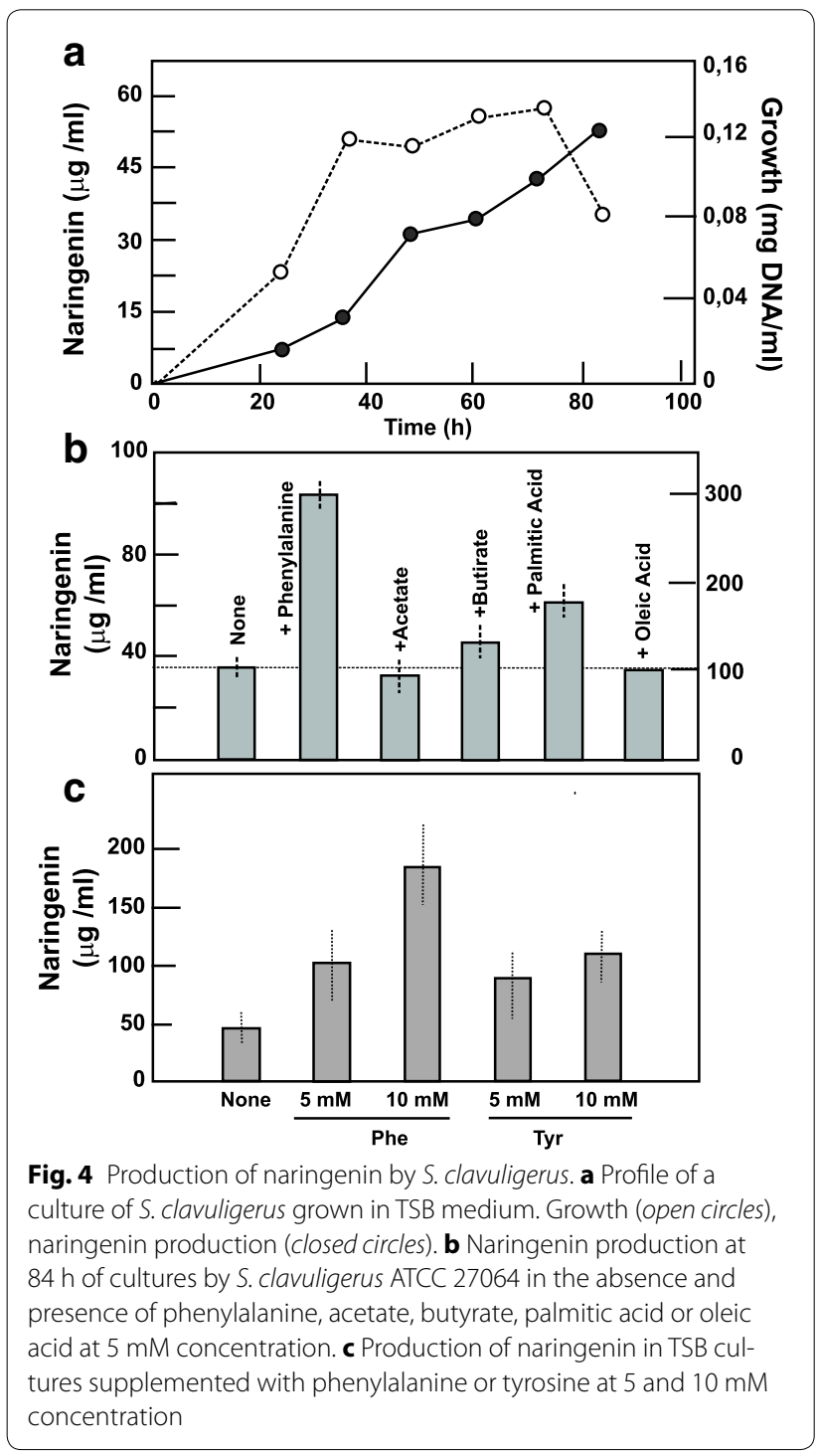

by 1.83 - and 2.32 -fold at 5 and $10 \mathrm{mM}$ concentration, respectively. The best production was observed by addition of phenylalanine, resulting in a naringenin increase of 2.16- and 3.7-fold respectively, at 5 and $10 \mathrm{mM}$ concentration (up to $184 \mu \mathrm{g} / \mathrm{ml}$ ). This concentration is much higher than the production obtained by a multistep engineered E. coli strain [14] indicating that S. clavuligerus ATCC 27064 is a good naringenin producer.

\section{Bioinformatic analysis of genes putatively encoding naringenin biosynthetic enzymes in the S. clavuligerus genome}

The putative pathway for naringenin biosynthesis (Fig. 1) requires the action of a naringenin chalcone synthase (Ncs) condensing the $p$-coumaroyl-CoA starting unit and three malonyl-CoA molecules. Therefore, using the sequence of Glycine max naringenin chalcone synthase (CAA38456) we searched S. clavuligerus genome for homologous genes using the BLAST Program. A single match corresponded to S. clavuligerus SCLAV_5492; this gene (named $n c s$ ) encodes a 351 amino acid protein with characteristics of type III polyketide synthases and a $29 \%$ identity (44 \% functionally conserved amino acids) along the entire sequence with G. max naringenin chalcone synthase, hereafter named Ncs. Interestingly, this chalcone synthase is closely related to type III PKSs of the RppA family occurring in many Streptomyces species (see "Discussion").

Inmediately upstream of ncs we found a gene, SCLAV_5491, encoding a 454 amino acid cytochrome $\mathrm{P}_{450}$ oxygenase that will be named hereafter $n c y P$. Genes encoding $\mathrm{P}_{450}$ oxygenases are frequently present in secondary metabolites gene clusters and we postulated that $n c y P$ might encode the enzyme required for the chalcone cyclization (see deletion and complementation experiments). This cytochrome $\mathrm{P}_{450}$ gene is more closely related to rppA-linked $\mathrm{P}_{450}$ 's in several Streptomyces species than to other $\mathrm{P}_{450}$ 's genes present in $S$. clavuligerus genome (see "Discussion").

Genes encoding proteins analogous to Ncs and NcyP are present with the same organization in S. coelicolor (SCO1206 and 1207), S. lividans (Sli_1485 and 1486) or S. griseus (SGR_6619 and 6620) while in S. venezuelae (SVEN_5366 and 5367) are located tail to tail, and in S. avermetilis (SAV_1171 and SAV_7131) are separated in the genome. The linkage of the $n c s$ and $n c y P$ genes may be relevant for efficient formation of naringenin (see "Discussion"). A tyrosine, at position 224, conserved in the $S$. clavuligerus Ncs protein, might be involved in the recognition of the first (starter) unit of p-coumaroil-CoA, or malonyl-CoA, as proposed in the RppA synthase of $S$. coelicolor $[18,19]$.

\section{Location of genes putatively involved in the formation of the 4-coumaroyl-CoA precursor}

Naringenin is formed from a 4-coumaric acid starting unit. In order to find genes encoding the enzyme required for 4-coumaroyl biosynthesis, the encP gene of Streptomyces maritimus, encoding a phenylalanine/tyrosine ammonia lyase [16], was used to search S. clavuligerus genome. One single matching gene, SCLAV_5457, was found. This gene, located $47.3 \mathrm{~kb}$ upstream of the $n c s-$ $n c y P$ cluster encodes a 559 amino acid protein with $37 \%$ amino acids identity (51\% conserved residues) to EncP along the whole sequence. This protein is related to TAL or PAL phenylalanine/tyrosine ammonia lyases and the gene will be named tal hereafter [20]. These enzymes are able to deamine phenylalanine to form cinnamic acid and tyrosine to produce coumaric acid. A short chain 
dehydrogenase/reductase encoded by SCLAV_5458, located downstream of the former gene may act on the double bond of the propenoyl side chain of 4-coumaric acid to reduce the double bond.

Activation of coumaric acid is likely to be mediated by the action of the aryl-CoA ligase encoded by SCLAV_3408. This last enzyme contains an ATP binding site (TGDIL, amino acids 386-390) for activation of the aromatic acid as an acyl-adenylate and is similar to coumaroyl-CoA ligases of plants and Penicillium chrysogenum [20-22] and to the benzoyl-CoA ligase of $S$. maritimus involved in the biosynthesis of enterocin [16].

\section{Deletion of S. clavuligerus ncs and ncyP genes blocks naringenin biosynthesis}

In order to elucidate if the ncs or $n c y P$ genes are involved in naringenin biosynthesis we deleted, separately, each of these genes using the Redirect procedure. Seven clones of $S$. clavuligerus $\Delta n c s:: a a c$ were obtained. The deleted strains showed the same pigmentation, aerial mycelium and spores formation as the parental strain. However, when tested for naringenin biosynthesis in TSB liquid cultures, production of naringenin was drastically reduced to about $9 \%$ of that in the parental strain, in the seven clones at $84 \mathrm{~h}$ cultures (Fig. 5).

Deletion of $n c y P$ produced two exconjugants designated as $S$. clavuligerus $\Delta n c y P:: a a c$. Both mutants produced only traces of naringenin, about $4 \%$ of the level of the parental strain indicating that $n c y P$ (but not other $\mathrm{P}_{450}$ oxygenases present in the cell) is strictly required for naringenin biosynthesis. Therefore, for the first time it is demonstrated that both genes, $n c s$ and $n c y P$, are involved in naringenin formation.

\section{Complementation of the ncs and ncyP genes in the deleted} mutants restores naringenin production

Streptomyces clavuligerus mutants $\Delta n c s:: a a c$ and $\Delta n c y P:: a a c$ were complemented with each of the deleted genes subcloned in the integrative plasmid pAV11 downstream of the anhydrotetracycline-inducible tcp 80 promoter. The complemented strains were grown in TSB medium in the presence of anhydrotetracycline $(10 \mu \mathrm{g} /$ $\mathrm{ml}$ ) to induce the $t c p 80$ promoter [23]. Production of naringenin was measured after $96 \mathrm{~h}$ of culture in all strains. S. clavuligerus ATCC 27064 produced $58 \mu \mathrm{g} /$ $\mathrm{ml}$. The control transformant with the integrated empty plasmid, S. clavuligerus [pAV11], produced a slightly lower naringenin level $(48 \mu \mathrm{g} / \mathrm{ml})$. The production in the deleted mutants and their controls transformed with the empty plasmid dropped to $2-7 \mu \mathrm{g} / \mathrm{ml}$ in the different strains. However, complementation of the ncs-deleted mutant resulted in restoration of naringenin production (to $55 \mu \mathrm{g} / \mathrm{ml}$ ) and complementation of the $n c y P$-deleted mutant resulted in naringenin concentrations of $42 \mu \mathrm{g} /$ $\mathrm{ml}$ (Fig. 5b). These results confirmed the involvement of $n c s$ and $n c y P$ in naringenin biosynthesis.
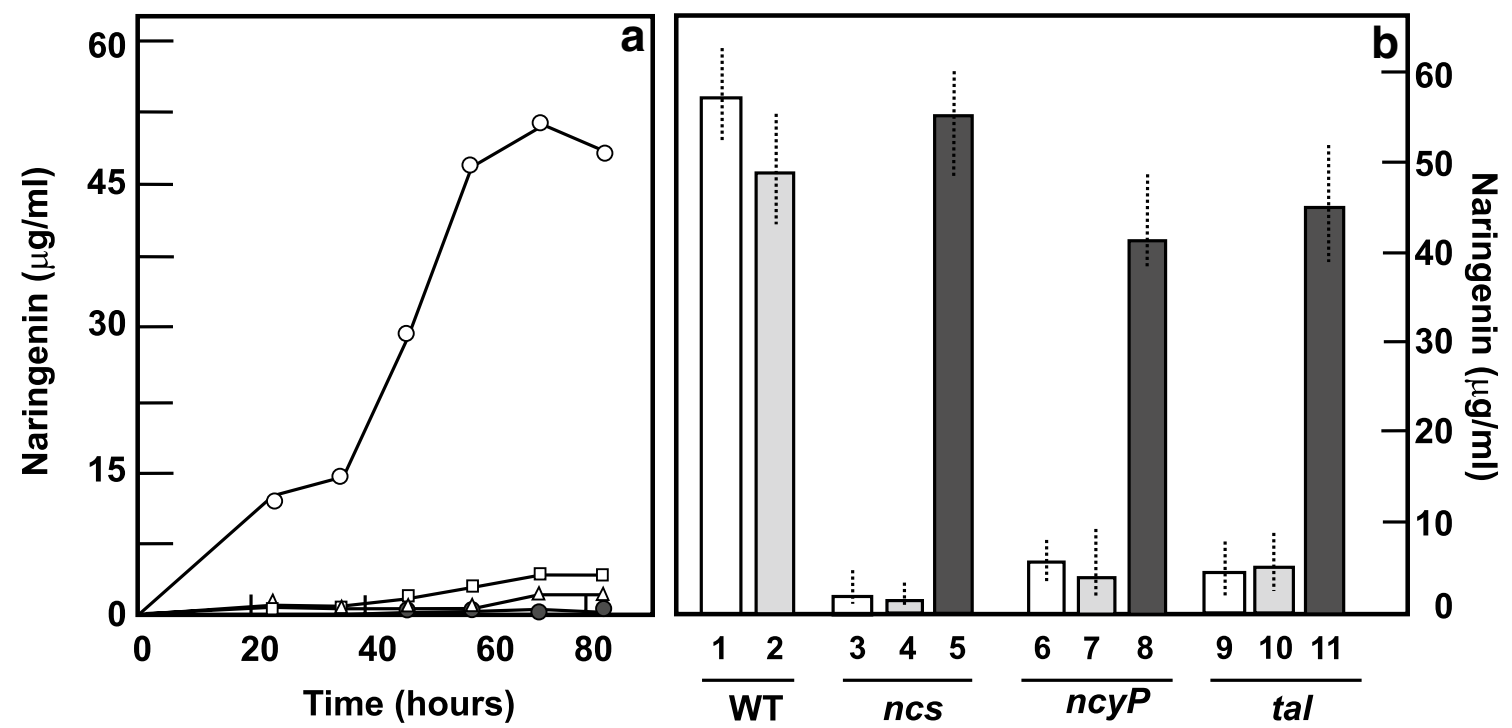

Fig. 5 Production of naringenin by S. clavuligerus deleted mutants and complemented strains. a Production of naringenin by S. clavuligerus 27064 (open circles), S. clavuligerus ncs::aac (closed circles), S. clavuligerus ncyP::aac (open triangles), S. clavuligerus tal::aac (open squares). b Production of naringenin by S. clavuligerus deleted and complemented strains at 96 h of growth. (1) S. clavuligerus ATCC27064, (2) S. clavuligerus [pAV1 1], (3) S. clavuligerus $\triangle n c s:: a a c$ (4) S. clavuligerus $\triangle$ ncs::aac [pAV11], (5) S. clavuligerus $\triangle n c s:: a a c$ [pAV11-ncs], (6) S. clavuligerus $\triangle$ ncyP::aac (7) S. clavuligerus $\triangle$ ncyP::aac [pAV11], (8) S. clavuligerus $\triangle$ ncyP::aac [pAV11-ncyP], 9) S. clavuligerus $\triangle$ tal::aac, (10) S. clavuligerus $\Delta$ tal::aac [pAV1 1], and (11) S. clavuligerus $\triangle$ tal::aac [pAV11-tal] 


\section{S. clavuligerus $\Delta$ tal::aac, deleted in the tal gene, does not produce naringenin but production is restored by complementation}

The tal gene, encoding a TAL/PAL enzyme was cloned from S. clavuligerus genomic DNA. Using oligonucleotides tal_F and tal_R a 1929 bp DNA fragment was amplified, sequenced and used to locate a cosmid containing the tal gene. Two cosmids D6-6 and D9-10 gave positive hibridization with the $1.9 \mathrm{~kb}$ DNA fragment. The sequencing of the insert of both cosmids showed that the tal gene was in a central position in cosmid D9-10 which was used for further studies. Seven apramycin resistant kanamycin sensitive clones, deleted in tal, were obtained by the Redirect technique. The seven clones were identical by restriction mapping, PCR amplification and sequencing confirming that the tal gene was substituted by the $a a c$ gene; the deleted strain was named $S$. clavuligerus $\Delta$ tal::aac. The tal mutants showed a normal behavior in sporulation and aerial mycelium formation in TSB and ME media. When they were grown in TSB liquid cultures the naringenin production at $96 \mathrm{~h}$ was lower than $6 \%$ in comparison to the wild type strain. Complementation of one of these mutants with the tal gene results in a transformant producing $46 \mu \mathrm{g} /$ $\mathrm{ml}$, which is $95 \%$ of the production of the control strain S. clavuligerus [pAV11] (Fig. 5b). This confirms that the ammonia lyase encoded by tal is involved in naringenin formation, probably by using tyrosine or phenylalanine as substrate(s) to form the 4-coumaric acid precursor unit. In this respect, $S$. clavuligerus is similar to monocotyledonous plants that are able to use a TAL to form directly $p$-coumaric acid and differs from dicotyledonous plant cells which form coumaric acid through a hydroxylation of trans-cinnamic acid by the enzyme cinnamate-4-hydroxylase. Our study showed that $S$. clavuligerus genome does not contain a cinnamate-4-hydroxylase.

\section{RT-PCR transcription analysis of the genes involved in naringenin biosynthesis}

Expression of $n c s$ and $n c y P$ in two different media (SA and TSB) was tested by RT-PCR at 24 and $48 \mathrm{~h}$ of culture. As shown in Fig. 6 a very weak amplification band of these two genes is observed in cultures grown in SA medium while a strong amplification signal was found in TSB medium what corroborates our initial results on production of naringenin in each of these media. Expression of these genes might be induced by soja-derived compounds in the triptic soja broth as reported for other $\mathrm{P}_{450}$ genes [24]. Using the oligonucleotides indicated in the Additional files 1, 2 an RT-PCR analysis was done for the intergenic $n c s-n c y P$ region. No amplification of the intergenic fragment was detectable (not shown) in RNA samples in which both $n c s$ and $n c y P$ were clearly amplified,

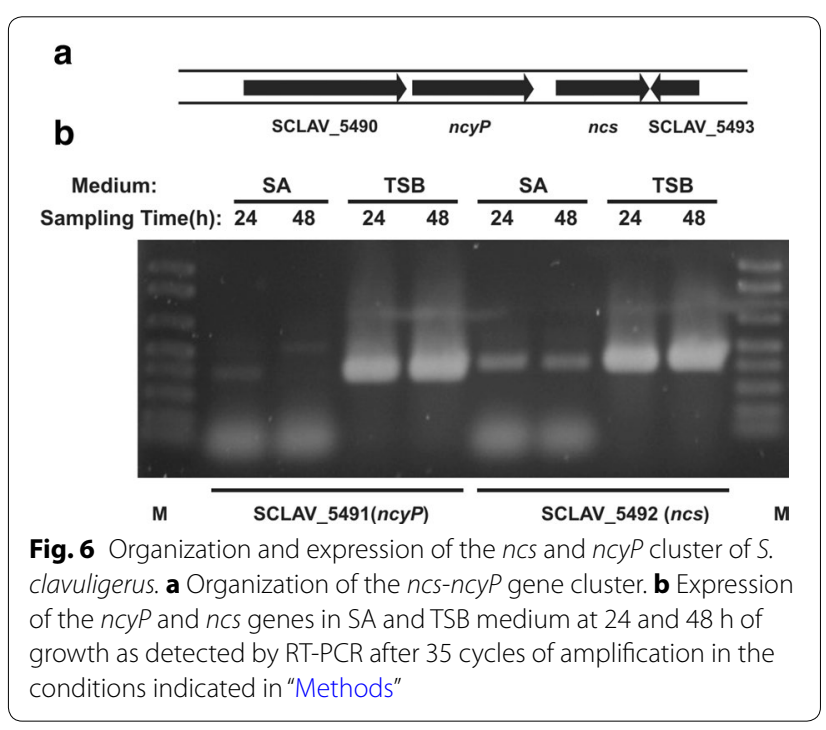

indicating that each of these genes was expressed as a monocistronic unit.

To determine whether the stimulatory effect observed by phenylalanine and tyrosine addition to the cultures was a precursor availability effect, or if it was due to an induction effect, quantification by qRT-PCR of the $n c s$, $n c y P$ and tal transcripts in control cultures and cultures supplemented with phenylalanine or tyrosine (each at 5 and $10 \mathrm{mM}$ concentration) was made. No significant differences in the transcription of the three genes was observed following phenylalanine or tyrosine addition, and only a very weak stimulation of the $n c s$ transcription was observed in the presence of phenylalanine. Therefore the stimulatory effect of these two aromatic amino acids on naringenin production is likely due to a precursor effect and not to induction of the naringenin biosynthetic genes.

\section{RppA versus Ncs: Streptomyces coelicolor forms tetrahydroxynaphthalene but does not produce naringenin}

Bacterial type III polyketide synthases are a group of PKSs that contain two KS domains and catalyze repeated condensations of malonyl/CoA units. They are similar to plant chalcone synthases [25] in amino acid sequence, although the plant chalcone synthases are usually larger enzymes. Some type III PKSs have been described in soil dwelling Streptomyces species and some related actinobacteria [26]. Several of these PKSs are involved in the production of red-brown pigments and consequently the encoding gene was named rppA [27-29]. The best known of these PKSs are the 1,3,6,8 tetrahydroxynaphtalene (THN) synthases of S. griseus and S. coelicolor encoded 
by rppA genes. In the formation of THN the RppA PKS uses malonyl-CoA as starter unit and three additional malonyl-CoA as elongation units [30]. In some Streptomyces species the THN is further oxidized to flaviolin [26]. Although S. clavuligerus Ncs and S. coelicolor RppA are related enzymes our study showed that $S$. clavuligerus does not produce red brown THN pigment and, additionally, the $\Delta n c s$ mutant culture broth remains of the same colour as the wild type cultures. Moreover, S. coelicolor A3(2), that is well known to synthesize THN does not produce naringenin when cultured in TSB medium under conditions in which $S$. clavuligerus produces this compound.

It was therefore of interest to know if $S$. coelicolor is able to produce naringenin when supplied, exogenously or endogenously, with $p$-coumaric acid. To test if the $S$. coelicolor RppA might be able to produce naringenin, two different experimental approaches were followed. First, S. coelicolor M145 cultures were supplemented with coumaric acid (5 mM). Second, S. coelicolor M145 was transformed with the S. clavuligerus tal gene (SCLAV_5457) expressed from the anhydrotetracyclin inducible tcp830 promoter in plasmid pAV11 to provide endogenous coumaric acid. Cultures of $S$. coelicolor [pAV11tal] were supplemented with phenylalanine, tyrosine or coumaric acid $(5 \mathrm{mM})$ and compared with cultures of $S$. coelicolor [pAV11] transformed with the empty plasmid, grown in the same conditions. None of this strategies resulted in detectable levels of naringenin production by $S$. coelicolor suggesting that the RppA chalcone synthase is unable to use in vivo efficiently $p$-coumaroyl-CoA instead of malonyl-CoA as starter unit.

\section{Discussion}

In plants and in S. clavuligerus, as shown in this article, the initial product of the Ncs is the naringenin chalcone, that plants usually converted later to flavonoids. Naringenin is synthesized by cyclization of a tetraketide formed by condensation of a $p$-coumaroyl-CoA starter and three malonyl-CoA elongation units (Fig. 1). The cyclization mechanism catalyzed by Ncs that leads to the formation of the pyrone ring of naringenin (Fig. 1) is different from the aromatization mechanism that results in the formation of THN, which involves a $\mathrm{C}-\mathrm{C}$ condensation with the free carboxyl group of the starter malonyl-CoA.

The $n c s-n c y P$ gene cluster and the tal gene, although located in two separate positions ( $47.3 \mathrm{~kb}$ apart) in the genome cooperate in the formation of the $p$-coumaric acid precursor unit and in the assembly of the naringenin molecule, an usual feature of genes for secondary metabolites that normally are clustered in Streptomyces.

The crystal structure of some CHSs [31,32] revealed an important role of the size of the cavity of the active center in the selection of the starter unit, and therefore, of the product of the pathway. This may explain the different products of NCS versus other related type III PKSs.

Streptomyces RppA's were hypothesized to have a small active center cavity that allowed only the entry of small acyl-CoA derivatives such as malonyl-CoA [33] different from plant chalcone synthases that use bulky starter units such as $p$-coumaroyl-, caffeyl- or trans-cinnamoyl-CoA.

The amino acid sequence of $S$. clavuligerus Ncs and the RppAs of $S$. avermitilis and S. coelicolor are very similar (76 and $74 \%$ identity, respectively). All these proteins posseses the four amino acid residues characteristics of chalcone synthases active center $\left(\mathrm{Cys}^{138}, \mathrm{Phe}^{188}, \mathrm{His}^{270}\right.$ and $\mathrm{Asn}^{303}$ in Ncs enzyme) [31]. The sequence of amino acids around the active center is different in Streptomyces and plant chalcone synthetases (Additional file 1: Figure S1), but $50 \%$ of these amino acids are conserved. Therefore, S. clavuligerus Ncs is a member of the RppA family and is phylogenetically closer to Streptomyces than to plant chalcone synthases in spite of giving a final plant typical product. However, S. clavuligerus does not produce significant amounts of THN or THN-derived pigments indicating that $p$-coumaroyl-derived naringenin is the major product of this enzyme. Furthermore, the $S$. clavuligerus $\triangle n c s$ mutant is not albino at difference of the $S$. griseus $\triangle r p p A$ mutant and does not change in colour with respect to its parental strain.

The $\mathrm{Tyr}^{224}$ in the entry channel of RppA's was proposed as the amino acid determining the selective use of malonyl-CoA as starter unit, although this hypothesis was later disputed by new evidence provided by a set of mutants in the Tyr ${ }^{224}$ amino acid [18]. Interestingly, the $S$. clavuligerus Ncs has a conserved Tyr at position 224 thus indicating that this amino acid is not strictly required for the entry of $p$-coumaroyl-CoA as starter unit of the polyketide, which is in agreement with the finding of Li et al. [18].

Linked to $n c s$ in S. clavuligerus genome is a $n c y P$ gene encoding a $\mathrm{P}_{450}$ oxygenase that is strictly required for naringenin biosynthesis. Similarly the $\mathrm{P}_{450}$ gene associated with rppA in S. griseus (known as rppB) is also required for THN formation [32, 34]. Our studies show that those two genes separatedly transcribed whereas in S. griseus they appear to be co-transcribed and translationally coupled, suggesting that both proteins are likely to form a functional complex. Cortés et al. [27] suggested that the RppA associated $\mathrm{P}_{450}$ has a role in cross-linking of THN molecules and this hypothesis was later supported by the study of strains overexpressing $r p p B$ that form dimers and trimmers of flavolin, the quinone derived from THN. Moreover, this $\mathrm{P}_{450}$ oxygenase has probably a role as a helper in the first cyclization of the tetraketides, both in THN and naringenin biosynthesis, what explains the 
essential role of these $\mathrm{P}_{450}$ oxygenases in the formation of $\mathrm{THN}$ and naringenin, that later proceed by different final cyclization processes.

\section{Tyrosine ammonia lyase and formation of $p$-coumaroyl-CoA}

Phenylalanine and tyrosine ammonia lyases belong to a family of aromatic amino acid ammonia lyases that are closely related to bacterial histidine ammonia lyases and aminomutases [35]. These enzymes catalyze the nonoxidative deamination of aromatic amino acids resulting in the formation of trans-p-coumaric acid when the substrate is L-tyrosine or trans-cinnamic acid when the substrate is phenylalanine. The biosynthesis of transcinnamic acid in dicotyledonous plants is catalyzed by a large PAL that works as a tetramer. A separate enzyme cinnamate 4-hydroxylase introduces the hydroxyl group at position 4 of the aromatic ring. In monocotyledonous plants both reactions are catalyzed by a single enzyme with TAL/PAL enzyme that uses both tyrosine and phenylalanine as substrates [36]. The bacterial aryl ammonia lyases are smaller (about 400 amino acids) tetrameric ammonia lyases [35]. Based on the crystal structure of the Rhodobacter histidine ammonia lyase and on the kinetics data of some mutant enzymes Louie and coworkers [36] proposed that $\mathrm{His}^{89}$ has an important role in this enzyme recognition of histidine or aromatic amino acids as substrates, since $\mathrm{His}^{89}$ forms hydrogen bridges with the substrates of the enzyme.

S. clavuligerus TAL, as the homologous enzyme of Saccharothrix spanaensis involved in the biosynthesis of saccharomicin [37], has a rather different amino terminal region when compared with the Rhodobacter enzyme, although six motifs described in the Rhodobacter enzyme to interact with the substrate are conserved to some degree (Fig. 7). The $\mathrm{H}^{89}$ present in one of these motifs in the Rhodobacter ammonia lyase corresponds to $\mathrm{Y}^{105}$ in $S$. clavuligerus TAL; this $\mathrm{Y}^{105}$ is conserved (or substituted by Phe) in a small group of actinobacteria which have ammonia lyases with 50-60 \% identity to $S$. clavuligerus TAL, while the bulk of Streptomyces ammonia lyases have about $40 \%$ identity with the $S$. clavuligerus enzyme, and differ in that they have an IVRSH sequence with serine at the corresponding 105 position. Using in vitro reactions with the Saccharothrix enzyme, that has a LIKYH domain carrying tyrosine [37] it has been clearly demonstrated that the product of this ammonia lyase is $p$-coumaric acid and not trans-cinnamic acid and the substrate used is L-tyrosine. Similar observations have been made for tyrosine amino mutase of Streptomyces globisporus that forms $p$-coumaric acid as an intermediate in the $\alpha$ - to $\beta$ - tyrosine isomerization and has $37 \%$ identity to the S. clavuligerus TAL.

Aromatic amino acid ammonia lyases were thought to contain a dehydroalanine prosthetic group derived from serine (in the Rhodobacter sphaeroides HAL protein) but later molecular genetics and spectrophotometric studies $[38,39]$ established that the prosthetic group is methylidene-imidazol-5-one (MIO). This molecule is formed by autocatalytic dehydration and cyclization of the internal tripeptide ala-ser-gly that in $S$. clavuligerus TAL corresponds to amino acids $A^{164}-S^{165}-G^{166}$ (Fig. 7). This tripeptide precursor of the prosthetic MIO group is present in all putative aromatic amino acid ammonia lyases and amino acid mutases in several Streptomyces species. The prosthetic group MIO of aromatic amino acid ammonia lyases is also present in the S. clavuligerus TAL protein as deduced from spectrophotometric studies [ÂlvarezÁlvarez, unpublished results). 
In summary, three enzymes Ncs, NcyP and Tal are involved in naringenin formation in S. clavuligerus, a strain that does not produce the THN pigment in spite of the similarity of Ncs to the S. coelicolor RppA protein.

\section{Methods}

\section{Strains and culture conditions}

The wild type strain, Streptomyces clavuligerus ATCC 27064 was used to purify naringenin. Strains S. clavuligerus $\Delta$ ncs::aac, S. clavuligerus $\Delta n c y P:: a a c$ and $S$. clavuligerus $\triangle$ tal::aac were obtained from the wild type strain by the REDIRECT Technique. Streptomyces coelicolor [pAV11-tal] and S. coelicolor [pAV11] derive from S. coelicolor M145 and were used to study possible naringenin production.

All the strains were grown at $28{ }^{\circ} \mathrm{C}$ in an orbital shaker incubator at $220 \mathrm{rpm}$ for $24 \mathrm{~h} \mathrm{in} 500 \mathrm{ml}$ baffled flasks containing $100 \mathrm{ml}$ of TSB medium. A $5 \%(\mathrm{v} / \mathrm{v})$ of this seed culture was used to inoculate TSB (trypticaseine broth) or SA (starch-asparragine) cultures grown in the same conditions [40]. All the cultures were made in triplicate flasks. The TSB was supplemented with tyrosine, phenylalanine or with organic acids (acetate, butirate, oleic acid, palmitic acid) as sodium salts, when required. Cultures of transformants strains were supplemented with kanamycin $(50 \mu \mathrm{g} / \mathrm{ml})$, anhydrotetracycline $(10 \mu \mathrm{g} /$ $\mathrm{ml})$, hygromycin $(50 \mu \mathrm{g} / \mathrm{ml})$, apramycin $(50 \mu \mathrm{g} / \mathrm{ml})$ or nalidixic acid $(25 \mu \mathrm{g} / \mathrm{ml})$ when required. Complex ME medium [41] was used for sporulation purposes.

\section{Naringenin purification by HPLC}

Naringenin was purified by HPLC using an Alliance 2695 HPLC with a refrigerated autosampler, a column furnace and a photodiode array detector (PDA) 2998 (Waters Corp.). Naringenin was quantified at its maximum absortion peak $(290 \mathrm{~nm})$. The system was equipped with a $3.5 \mu \mathrm{m}$ particle size XBridge ${ }^{\mathrm{TM}} \mathrm{C} 18(2.1 \times 150 \mu \mathrm{m}$, Waters Corp.) column.

The culture samples were centrifuged at 13,000 rpm for $5 \mathrm{~min}$ and the supernatants filtered through sterile $0.2 \mu \mathrm{m}$ polyethersulphone (PES) syringe filters (VWR, Intl.). The whole sample $(100 \mathrm{ml})$ was loaded in multiple injections. HPLC conditions were as follows: column temperature $40{ }^{\circ} \mathrm{C}$; the flow was kept at $0.32 \mathrm{ml} / \mathrm{min}$ from 0 to $2 \mathrm{~min}$ and then increased gradually to reach $0.40 \mathrm{ml} /$ min at $10 \mathrm{~min}$. A mixture of Milli Q water with $0.01 \%$ trifluoroacetic acid (A) and acetonitrile (B) were used as solvent with the following program: $0-2$ min increase from 20 to $30 \%$ B and kept at $30 \%$ B thereafter. The peak eluting at $6 \mathrm{~min}$ from repeated injections was collected and pooled and the solvent from the pooled preparation was evaporated under low pressure (SpeedVac Savant Sc110, Thermo Sci.). The concentrated solution was lyophilized and kept at $-20{ }^{\circ} \mathrm{C}$ until NMR analysis and identification. Pure naringenin and genistein (Sigma Ch. Co.) were used as standards.

\section{NMR analysis}

NMR spectra were recorded in metanol- $\mathrm{d}_{4}$ at room temperature using a Bruker WM 500 spectrometer $\left[500 \mathrm{MHz}\left({ }^{1} \mathrm{H}\right.\right.$ NMR) and $\left.125 \mathrm{MHz}\left({ }^{13} \mathrm{C} \mathrm{NMR}\right)\right]$. Chemical shifts are given on the $\delta$-scale and were referenced to the solvent $(\delta \mathrm{c}=49.0 \mathrm{ppm})$ and to the TMS as internal standard. The pulse programs of the following $2 \mathrm{D}$ experiments were taken from the Bruker software library and the parameters were as follows: $500 / 125 \mathrm{MHz}$ gradientselected HMQC spectra [1]: relaxation delay $\mathrm{D}_{1}=1.5 \mathrm{~s}$; $500 / 125 \mathrm{MHz}$ gradient-selected HMBC spectra [1]: relaxation delay $D_{1}=1.5 \mathrm{~s}$; evolution delay $D_{2}=3.33 \mathrm{~ms}$; delay for evolution of long range coupling $\mathrm{D}_{6}=60 \mathrm{~ms}$. $500 \mathrm{MHz}$ gradient-selected ${ }^{1} \mathrm{H},{ }^{1} \mathrm{H}$ COSY spectra [2]: relaxation delay $\mathrm{D}_{1}=1.5 \mathrm{~s} ; 90^{\circ}$ pulse for ${ }^{1} \mathrm{H}$.

\section{Mass spectrometry}

The mass spectra were determined by HPLC-MS (Agilent Technologies). Using a HP-1100 Series HPLC and MS: 6120 Quadrupole LC/MS.

\section{Extraction and purification of RNA}

RNA was extracted from $1.5 \mathrm{ml}$ samples taken at 24 and $48 \mathrm{~h}$ of growth in TSB and SA media. Two volumes of RNA-protect (Qiagen) were added to the samples. The commercial system Qiagen, RNAeasy was used following the manufacturer instructions as described previously [40].

\section{RT-PCR}

For RT-PCR amplification the final $20 \mu \mathrm{l}$ reaction contained RNA (200-300 ng), reaction mixture $1 \times, 0.5 \mathrm{mM}$ each oligonucleotide (see Additional file 2: Table S1); 5 \% DMSO; SuperScriptTM II Reverse Transcriptase and Platinum $^{\circledR}$ Taq (Invitrogen) 1-2 units. Control reactions were done with the same procedure but only with Platinum $^{\circledR}$ Taq. Amplification conditions: cDNA was formed in a $50{ }^{\circ} \mathrm{C} 30 \mathrm{~min}$ cycle. Then, an initial $94{ }^{\circ} \mathrm{C} 2 \mathrm{~min}$ denaturation cycle was followed by $25-3530$-s cycles at $95^{\circ} \mathrm{C}$. A final extension cycle of $10 \mathrm{~min}$ at $72{ }^{\circ} \mathrm{C}$ reaction was allowed.

\section{Construction of the complemented strains}

In order to complement the $n c s, n c y P$ and tal-deleted strains, each of those genes was obtained by PCR amplification, sequenced to confirm their integrity and subcloned downstream of the anhydrotetracycline-inducible tcp-830 promoter in the EcoRV site of the conjugativeintegrative vector pAV11 [23]. The constructions were 
introduced in E.coli ET12567/pUZ8002, and then were transferred to the adequate Streptomyces strain by conjugation. Hygromycin resistant exconjugants were tested by PCR to confirm the presence of the complementing genes.

\section{Additional files}

Additional file 1: Figure S1. A) Conserved sequences around the key amino acids located in chalcone synthases active center. The numbers given in the sequence correspond to S. clavuligerus Ncs, where the key amino acids are $\mathrm{Cys}^{138}$, Phe ${ }^{188}, \mathrm{His}^{270}$ and $\mathrm{Asn}^{303}$. B) Phylogenetic three of plant and Streptomyces chalcone synthases. The origin of the sequences used is indicated at the right side. Numbers at the far right side correspond to the genetic distance index.

Additional file 2: Table S1. Oligonucleotides used in this work.

\section{Authors' contributions}

RA fermented the supplemented strains, analyzed the transcription of the genes and cloned, deleted and complemented the ncs and tal genes. AB cloned, deleted and complemented the ncyP gene. AR identified naringenin by $L C$ and NMR. SA purified the naringenin and performed the HPLC analysis. JFM and PL directed the experimental work and write the manuscript. All authors read and approved the final manuscript.

\section{Author details}

${ }^{1}$ Microbiology Section, Department of Molecular Biology, Faculty of Biology and Environmental Sciences, Vegazana Campus, University of León, León 24071, Spain. ${ }^{2}$ Institute of Biotechnology, INBIOTEC, Av. Real 1, León 24006, Spain. ${ }^{3}$ Organic Chemistry Department, University Autónoma of Madrid, Cantoblanco, 28049 Madrid, Spain.

\section{Acknowledgements}

This work was supported by Grant BIO2012-34723 from the Spanish Ministry of Economy and Competitivity. R. Álvarez-Álvarez received a FPU fellowship from the Spanish Ministry of Education, Culture and Sports.

\section{Competing interests}

The authors declares that they have no any competing interests in the manuscript.

Received: 15 July 2015 Accepted: 29 October 2015

Published online: 09 November 2015

\section{References}

1. Baggaley KH, Brown AG, Schofield CJ. Chemistry and biosynthesis of clavulanic acid and other clavams. Nat Prod Rep. 1997;14:309-33.

2. Pérez-Redondo R, Santamarta I, Bovenberg R, Martín JF, Liras P. The enigmatic lack of glucose utilization in Streptomyces clavuligerus is due to inefficient expression of the glucose permease gene. Microbiology. 2010;156:1527-37.

3. Liras P, Demain AL. Enzymology of $\beta$-lactam compounds with cephem structure produced by actinomycete. In: Hopwood D, editor. Methods in enzymology, vol. 458. Burlington: Academic Press; 2009. p. 401-29.

4. Jensen SE. Biosynthesis of clavam metabolites. J Ind Microbiol Biotechnol. 2012;39:1407-19.

5. Li B, Walsh CT. Identification of the gene cluster for the dithiolopyrrolone antibiotic holomycin in Streptomyces clavuligerus. Proc Natl Acad Sci USA. 2010;107:19731-5.

6. Robles-Reglero V, Santamarta I, Álvarez-Álvarez R, Martín JF, Liras P. Transcriptional analysis and proteomics of the holomycin gene cluster in overproducer mutants of Streptomyces clavuligerus. J Biotechnol. 2013;163:69-76.
7. Medema MH, Trefzer A, Kovalchuk A, vandenBerg M, Müller U, Heijne W, Wu L, Alam MT, Ronning CM, Nierman WC, Bovenberg RA, Breitling R, Takano $\mathrm{E}$. The sequence of a 1.8-Mb bacterial linear plasmid reveals a rich evolutionary reservoir of secondary metabolic pathways. Genome Biol Evol. 2010;2:212-24.

8. Song JY, Jeong H, Yu DS, Fischbach MA, Park HS, Kim JJ, Seo JS, Jensen SE, Oh TK, Lee KJ, Kim JF. Draft genome sequence of Streptomyces clavuligerus NRRL 3585, a producer of diverse secondary metabolites. J Bacteriol. 2010;192:6317-8.

9. Ochi K, Hosaka T. New strategies for drug discovery: activation of silent or weakly expressed microbial gene clusters. Appl Microbiol Biotechnol. 2013;97:87-98.

10. Martín JF, Liras P. Novel antimicrobial and other bioactive metabolites obtained from silent gene clusters. In: Sanchez S, Demain AL, editors. Antibiotics current innovations and future trends. Nordfolk: Caister Academic Press; 2014. p. 275-92.

11. Schröder J. Probing plant polyketide biosynthesis. J Nat Struct Biol. 1999;6:714-6.

12. Cavia-Saiz M, Busto MD, Pilar-Izquierdo MC, Ortega N, Pérez-Mateos M, Muñíz P. Antioxidant properties, radical scavenging activity and biomolecule protection capacity of flavonoid naringenin and its glycoside naringenin: a comparative study. J Sci Food Agric. 2010;90:1238-44.

13. Jagetia A, Jagetia GC, Jha S. Naringin, a grapefruit flavanone, protects V79 cells against the bleomycin-induced genotoxicity and decline in survival. J Appl Toxicol. 2007;27:122-32.

14. Wu J, Zhou T, Du G, Zhou J, Chen J. Modular optimization of heterologous pathways for de novo synthesis of (2S)-naringenin in Escherichia coli. Plos One. 2014;9:e101492.

15. Kyndt JA, Meyer TE, Cusanovich MA, Van Beeumen JJ. Characterization of a bacterial tyrosine ammonia lyase, a biosynthetic enzyme for the photoactive yellow protein. FEBS Lett. 2002;512:240-4.

16. Xiang L, Moore BS. Inactivation, complementation, and heterologous expression of enc $P$, a novel bacterial phenylalanine ammonia-lyase gene. J Biol Chem. 2002;277:32505-9.

17. Zhang XF, Hung TM, Phuong PT, Ngoc TM, Min B-S, Song K-S, Seong YH, Bae KH. Anti-inflammatory activity of flavonoids from Populus davidiana. Arch Pharm Res. 2006;29(12):1102-8.

18. Li S, Grüschow S, Dordik J, Sherman DH. Molecular analysis of the role of tyrosine 224 in the active site of Streptomyces coelicolor RppA, a bacterial Type III polyketide synthase. J Biol Chem. 2007;282:12765-72.

19. Appert C, Logemann E, Hahlbrock K, Schmid J, Amrhein N. Structural and catalytic properties of the four phenylalanine ammonia-lyase isoenzymes from parsley. Eur J Biochem. 1994;225:491-9.

20. Schneider K, Hövel K, Witzel K, Hamberger B, Schomburg D, Kombrink E, Stuible HP. The substrate specificity-determining amino acid code of 4-coumarate:CoA ligase. Proc Natl Acad Sci USA. 2003;100:8601-6.

21. Lamas-Maceiras M, Vaca I, Rodríguez E, Casqueiro J, Martín JF. Amplification and disruption of the phenylacetyl-CoA ligase gene of Penicilium chrysogenum encoding an aryl-capping enzyme that supplies phenylacetic acid to the isopenicillin $\mathrm{N}$-acyltransferase. Biochem J. 2006;395:147-55

22. Martín JF, Ullán RV, García-Estrada C. Role of peroxisomes in the biosynthesis and secretion of $\beta$-lactams and other secondary metabolites. J Ind Microbiol Biotechnol. 2012;39:367-82.

23. Rodríguez-García A, Combes P, Pérez-Redondo R, Smith MC, Smith MC. Natural and synthetic tetracycline-inducible promoters for use in the antibiotic-producing bacteria Streptomyces. Nucleic Acids Res. 2005;33:e87.

24. Sariaslani FS, Kunz DA. Induction of cytochrome P-450 in Streptomyces griseus by soybean flour. Biochem Biophys Res Commun. 1986;141:405-10.

25. Austin MB, Noel JP. The chalcone synthase superfamily of type III polyketide synthases. Nat Prod Rep. 2003;20:79-110.

26. Zeng J, Decker R, Zhan J. Biochemical characterization of a type III polyketide biosynthetic gene cluster from Streptomyces toxytricini. Appl Biochem Biotechnol. 2012;166:1020-33.

27. Cortés J, Velasco J, Foster G, Blaackaby AP, Rudd BAM, Wilkinson B. Identifcation and cloning of a type III polyketide synthase required for diffusible pigment biosynthesis in Saccharopolyspora erythraea. Mol Microbiol. 2002;44:1213-24.

28. Ikeda K, Masujima T, Suzuki K, Sugiyama M. Cloning and sequence analysis of the highly expressed melanin-synthesising gene operon 
from Streptomyces castaneoglobisporus. Appl Microbiol Biotechnol. 1996;4:80-5.

29. Funa N, Funabashi M, Ohnishi Y, Horinouchi S. Biosynthesis of hexahydroxyperylenequinone melanin via oxidative aryl coupling by cytochrome P-450 in Streptomyces griseus. J Bacteriol. 2005;187:8149-55.

30. Funa N, Ohnishi Y, Fujii I, Shibuya M, Ebizuka Y, Horinouchi S. A new pathway for polyketide synthesis in microorganisms. Nature. 1999;4000:897-9.

31. Ferrer JL, Jez JM, Bowman ME, Dixon RA, Noel JP. Structure of chalcone synthase and the molecular basis of plant polyketide biosynthesis. Nat Struct Biol. 1999;6:775-84.

32. Jez JM, Noel JP. Mechanism of chalcone synthase. pKa of the catalytic cysteine and the role of the conserved histidine in a plant polyketide synthase. J Biol Chem. 2000;275:39640-6.

33. Funa N, Ohnishi Y, Ebizuka Y, Horinouchi S. Properties and substrate specificity of RppA, a chalcone synthase-related polyketide synthase in Streptomyces griseus. J Biol Chem. 2002;277:4628-35.

34. Ueda K, Kim KM, Beppu T, Horinouchi S. Overexpression of a gene cluster encoding a chalcone synthase-like protein confers redbrown pigment production in Streptomyces griseus. J Antibiot. 1995;48:638-46.

35. Louie GV, Bowman ME, Moffitt MC, Baiga TJ, Moore BS, Noel JP. Structural determinants and modulation of substrate specificity in phenylalaninetyrosine ammonia-lyases. Chem Biol. 2006;13:1327-38.
36. Rosler J, Krekel F, Amrhein N, Schmid J. Maize phenylalanine ammonialyase has tyrosine ammonia-lyase activity. Plant Physiol. 1997;113:175-9.

37. Berner M, Krug D, Bihlmaier C, Vente A, Müller R, Bechthold A. Genes and enzymes involved in caffeic acid biosynthesis in the actinomycete Saccharothrix espanaensis. J Bacteriol. 2006;188:2666-73.

38. Schwede TF, Rétey J, Schulz GE. Crystal structure of histidine ammonialyase revealing a novel polypeptide modification as the catalytic electrophile. Biochemistry. 1999;38:5355-61.

39. Röther D, Merkel D, Rétey J. Spectroscopic evidence for a 4-methylidene imidazol-5-one in histidine and phenylalanine ammonia-lyases. Angew Chem Int Ed Engl. 2000;39:2462-4.

40. Santamarta I, López-García MT, Kurt A, Nárdiz N, Álvarez-Álvarez R, PérezRedondo R, Martín JF, Liras P. Characterization of DNA-binding sequences for CcaR in the cephamycin-clavulanic acid supercluster of Streptomyces clavuligerus. Mol Microbiol. 2011;81:968-81.

41. Sánchez L, Braña A. Cell density influences antibiotic biosynthesis in Streptomyces clavuligerus. Microbiology. 1996;142:1209-20.

\section{Submit your next manuscript to BioMed Central and take full advantage of:}

- Convenient online submission

- Thorough peer review

- No space constraints or color figure charges

- Immediate publication on acceptance

- Inclusion in PubMed, CAS, Scopus and Google Scholar

- Research which is freely available for redistribution

Submit your manuscript at

www.biomedcentral.com/submit

() BioMed Central 